\title{
Appendix
}

Table A1: 2001-2008 NASS-CDS crashes with GAD = B and 1992-2008 model year GM vehicles with baseline or high retention seats ( $\mathrm{n}$ : sample size, se: standard error.

\begin{tabular}{ccccccc} 
& \multicolumn{3}{c}{ Baseline seats } & \multicolumn{3}{c}{ High Retention seats } \\
MAIS & $\mathbf{n}$ & $\mathbf{n}$ & se & $\mathbf{n}$ & $\mathbf{n}$ & se \\
& unwgt & wgt & wgt & unwgt & wgt & wgt \\
0 & 151 & 92,504 & 15,072 & 118 & 58,303 & 9,939 \\
1 & 228 & 119,751 & 41,564 & 167 & 64,310 & 13,455 \\
2 & 12 & 2,112 & 1,253 & 11 & 2,494 & 902 \\
3 & 9 & 248 & 126 & 1 & 24 & 24 \\
$4+$ & 4 & 988 & 840 & 3 & 528 & 349 \\
$4+F$ & 19 & 1,862 & 1,070 & 7 & 616 & 353 \\
F & 15 & 874 & 567 & 4 & 88 & 30 \\
Unknown & 24 & 3,655 & 1,583 & 20 & 10,559 & 4,581 \\
Total & 443 & 220,132 & 53,809 & 324 & 136,306 & 21,131
\end{tabular}




\section{Appendix A2}

2001-9-154A: Vehicle 1 (case vehicle) was a 2002 Chevrolet Malibu. It was proceeding westbound on a three lane divided highway in an unspecified lane approaching a hillcrest. Figure A1 shows the path of Vehicle 1 and photos of the impact damage and interior. The roadway was dark but straight and dry. For unknown reasons, the vehicle departed the roadway to the left and struck a guardrail in the grass median (event 1: front plane, 12 o'clock PDOF). The Vehicle continued along the guardrail, sideswiping it. The vehicle came back out into the roadway into lane 1 on the other side of the hillcrest. Vehicle 2 (1992 Honda Accord) was also on this highway, in lane 1. It came over the hillcrest and struck Vehicle 1 (event 2: front to rear - severe underride, 7 o'clock PDOF). The vehicles were both towed due to damage. The final rest positions of the vehicles are uncertain. Occupants 2 (case occupant) and 3 of Vehicle 1 were ejected and killed. Occupant 2 landed in the left shoulder of the opposite lanes of traffic and Occupant 3 landed in lane 2. Both drivers of the vehicles were transported with possible injuries.

Occupants 2 (case occupant) was a 19 year old male unbelted in the right-front passenger seat. The NASS investigators indicated the front-passenger seat was deformed by the occupant. Photos of the vehicle interior show a slight counterclockwise twist with the inboard side of the seat deformed rearward. The $2^{\text {nd }}$ impact in the sequence was to the rear with a PDOF of 7 o'clock and delta $V$ of $30 \mathrm{mph}$. The investigators indicated ejection out the rear window along a path up the rear seatback (center, right photo). There were no other details of the source or type of injury related to the ejection and ground impact.
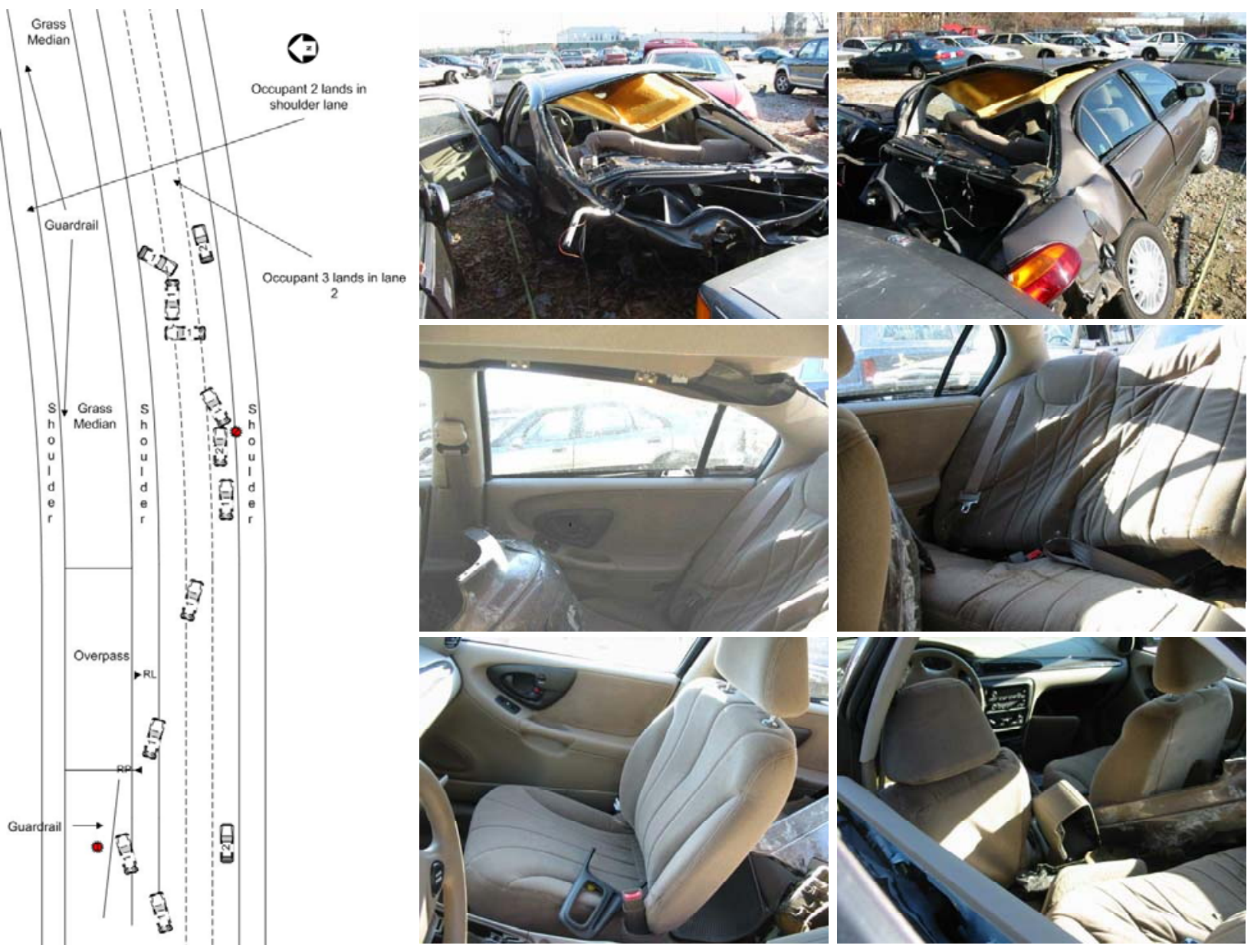

Figure A1: Vehicle path and photos for case 2001-9-154A. 
2002-73-157B: This three-vehicle nighttime crash occurred at 21:07 on a rural, four-lane US Route roadway that had a center painted paved area that separated the north/south travel lanes. The width of

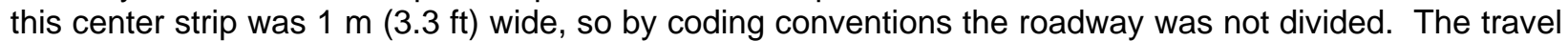
lanes were asphalt and they were dry, straight and level. There was no overhead light in this residential area. The posted legal speed limit was $55 \mathrm{mph}$. Vehicle 1 was a $1998 \mathrm{Chevy} \mathrm{S-10} \mathrm{pickup} \mathrm{truck.} \mathrm{Vehicle}$ 1 was traveling south in the number two travel lane and was occupied by the driver only.

Vehicle 2 (case vehicle) was a 1998 Pontiac Grand Prix. Figure A2 shows the path of Vehicle 2 and photos of the impact damage and interior. Vehicle 1 initiated the crash sequence. Its front impacted the rear of Vehicle 2, which was traveling south in the second travel lane and had slowed to a stop to make a left turn across the two opposite direction lanes onto a residential roadway. The driver and right-front passenger (case occupant) occupied vehicle 2.

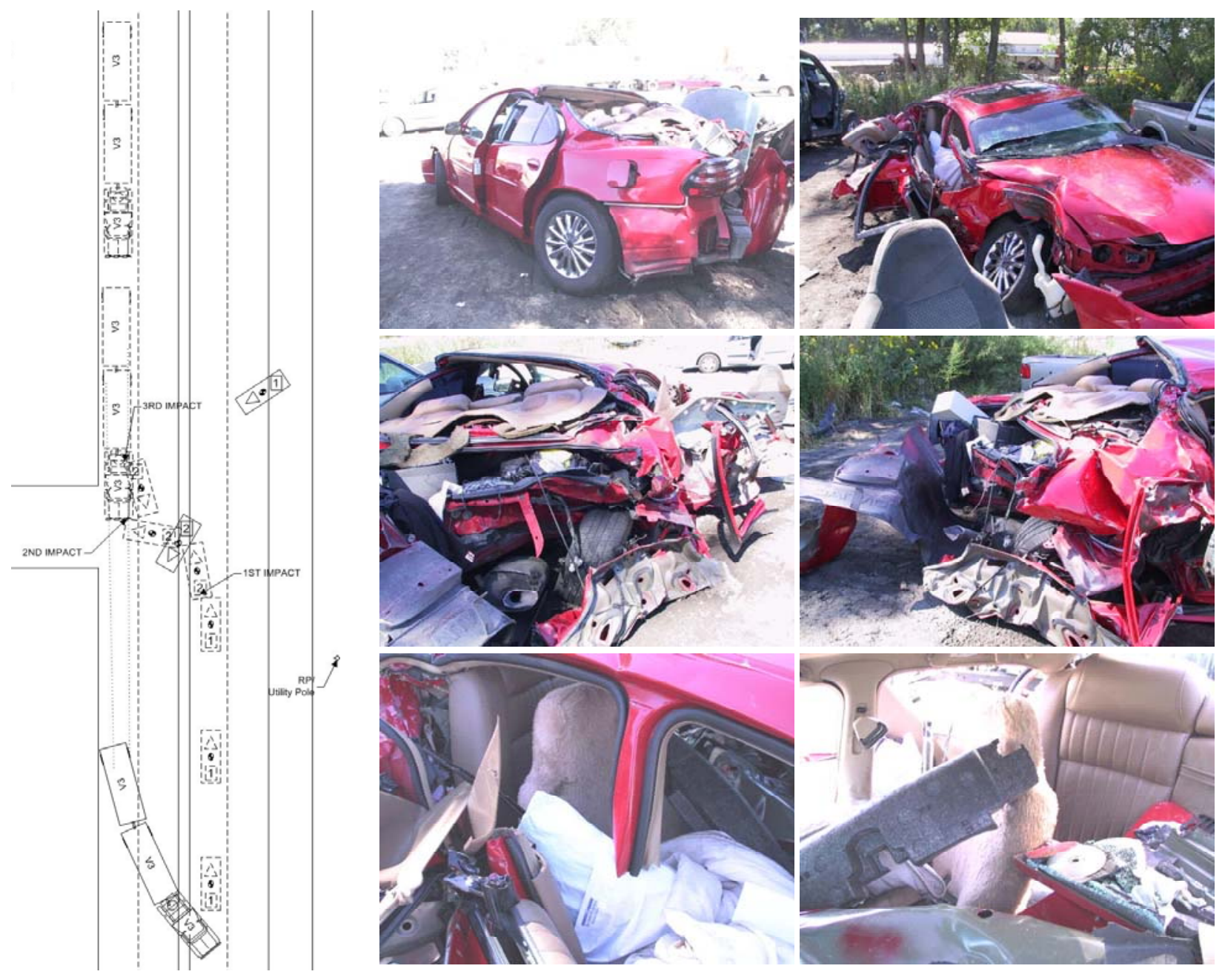

Figure A2: Vehicle path and photos for case 2002-73-157B.

Vehicle 3 was a 2002 Volvo tractor pulling two trailers; one was a semi and the other a full trailer connected by a C-dolly. The first semi-trailer was loaded with US mail weighing 7,069 $\mathrm{kg}(15,552 \mathrm{lb})$. The second full trailer was also loaded with US mail weighing $7,003 \mathrm{~kg}(15,407 \mathrm{lb})$. Vehicle 3 was traveling northbound on same roadway in the number one travel lane. Vehicle 1's front impacted vehicle 2's back while Vehicle 2 was stationary. Vehicle 2 was then pushed towards Vehicle 3 in a counterclockwise rotation. Vehicle 3's front impacted Vehicle 2's right side. Vehicle 2 continued to rotate in a counterclockwise motion and its right rear impacted vehicle 3's left side. Vehicle 1 came to final rest 
facing northeast in the first southbound lane after rotating in a counterclockwise motion. Vehicle 2 came to final rest facing north-northeast at about $20 \mathrm{deg}$ in the second northbound lane. Vehicle 3 and its trailers came to a final rest facing northwest covering both northbound lanes. Vehicle 1's restrained driver was transported and released with minor abrasions and contusions. Vehicle 1's airbags deployed during the first impact. Vehicle 2's driver was transported and released with minor facial abrasion and contusion. The airbags did not deploy in this vehicle. The right-front passenger of Vehicle 2 (case occupant) was taken by helicopter to a trauma center and expired due to multiple head trauma eight hours later. Vehicle 3's driver received a knee sprain but was not treated. All three vehicles were towed from the scene due to damage.

The right-front occupant (case occupant) in the 1998 Pontiac Grand Prix was a 22 year old unbelted female. She experienced partial ejection in the collision and her head received severe injuries including skull fractures, hemorrhages of the brain. The source for the head injuries was exterior of the vehicle and related to the partial ejection. She also experienced lung lacerations and contusions, and contusions of the skin on her upper and lower extremities. The NASS investigators indicated the front-passenger seat was deformed by the occupant. Photos of the vehicle interior show minimal deformation of the right-front passenger seat. The $1^{\text {st }}$ impact in the collision sequence was to the rear (GAD B) and was the most severe. The PDOF was 6 o'clock and delta $\vee$ of $32 \mathrm{mph}$.
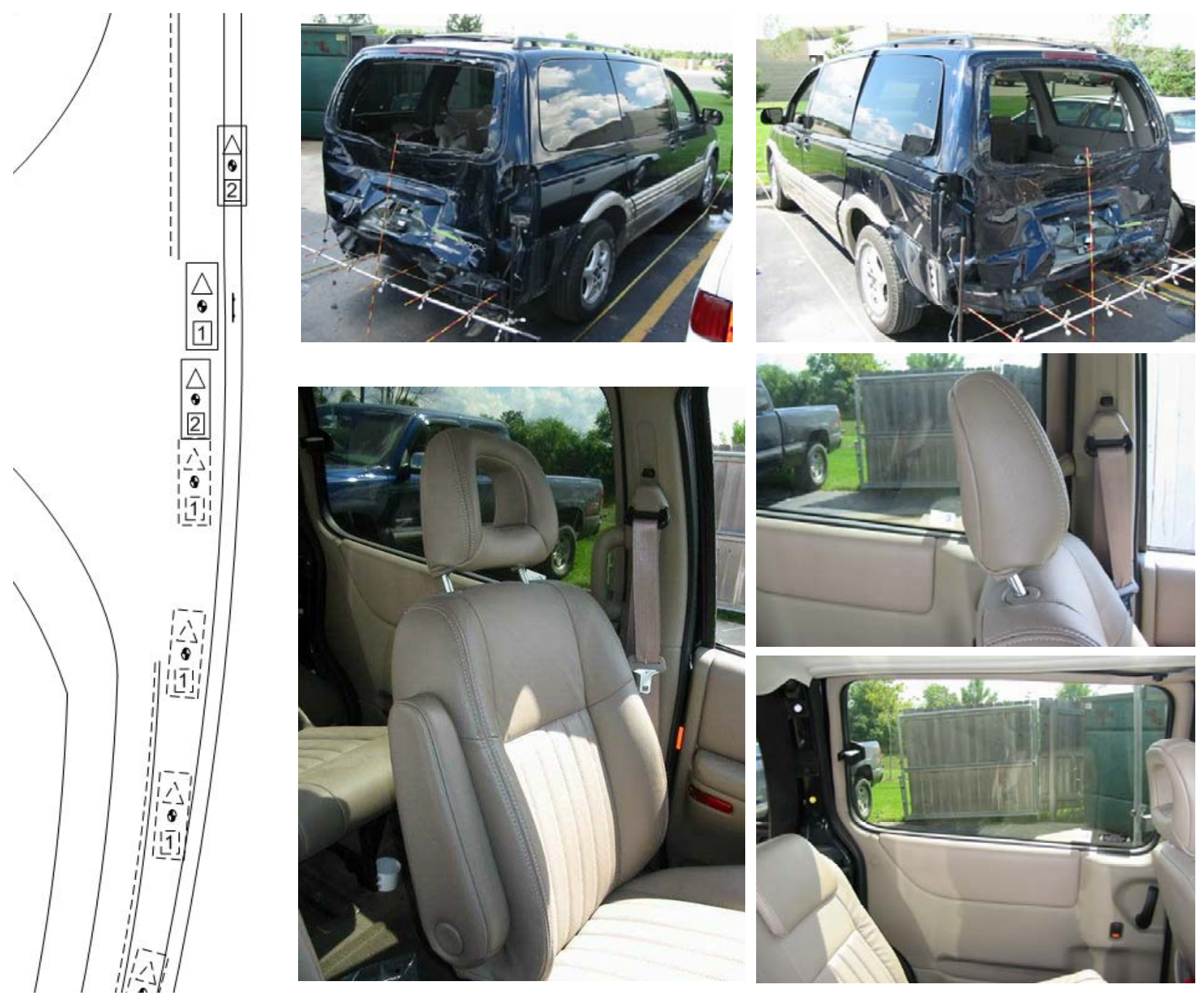

Figure A3: Vehicle path and photos for case 2002-12-115A. 
2002-12-115A: Vehicles 1 and 2 were heading north on a 2 lane, 2-way, dry, asphalt roadway that curved left. Vehicle 2 (case vehicle) was a 2001 Pontiac Montana. It was stopped at an intersection to make a left hand turn. Figure A3 shows the path of Vehicle 2 and photos of the impact damage and interior. Vehicle 1 was a 2000 Chevrolet G1500 Express van. It came around the curve and the front of the vehicle contacted the back of Vehicle 2 causing moderate damage to both vehicles. Both vehicles were towed due to damage. The driver of vehicle 1 sought no treatment for minor injuries. All occupants in vehicle 1 were wearing their safety belts and the frontal air bags did deploy. No injuries were sustained by any of the child occupants in V1.

The driver of vehicle 2 (case occupant) was a 72 year old female, who was wearing her lap and shoulder belts. The vehicle was equipped with front and side impact air bags, none of which deployed in this rearward impact. She was transported to a local trauma center where she later died of her injuries. The most severe injury was a thoracic spine fracture-dislocation at T7-T8 with a large gapping tear of the ligaments between the vertebral bodies with the spinal cord visible. She had bilateral rib fractures to the left ribs 2-5, 7 and 8 and on the right ribs 2-9 with a right hemo-pneumothorax. The NASS investigator associated the injuries with the seatback. Photos of the vehicle interior show minimal deformation of the driver seat. The rear impact had a PDOF of 6 o'clock and delta $\vee$ of $14 \mathrm{mph}$.

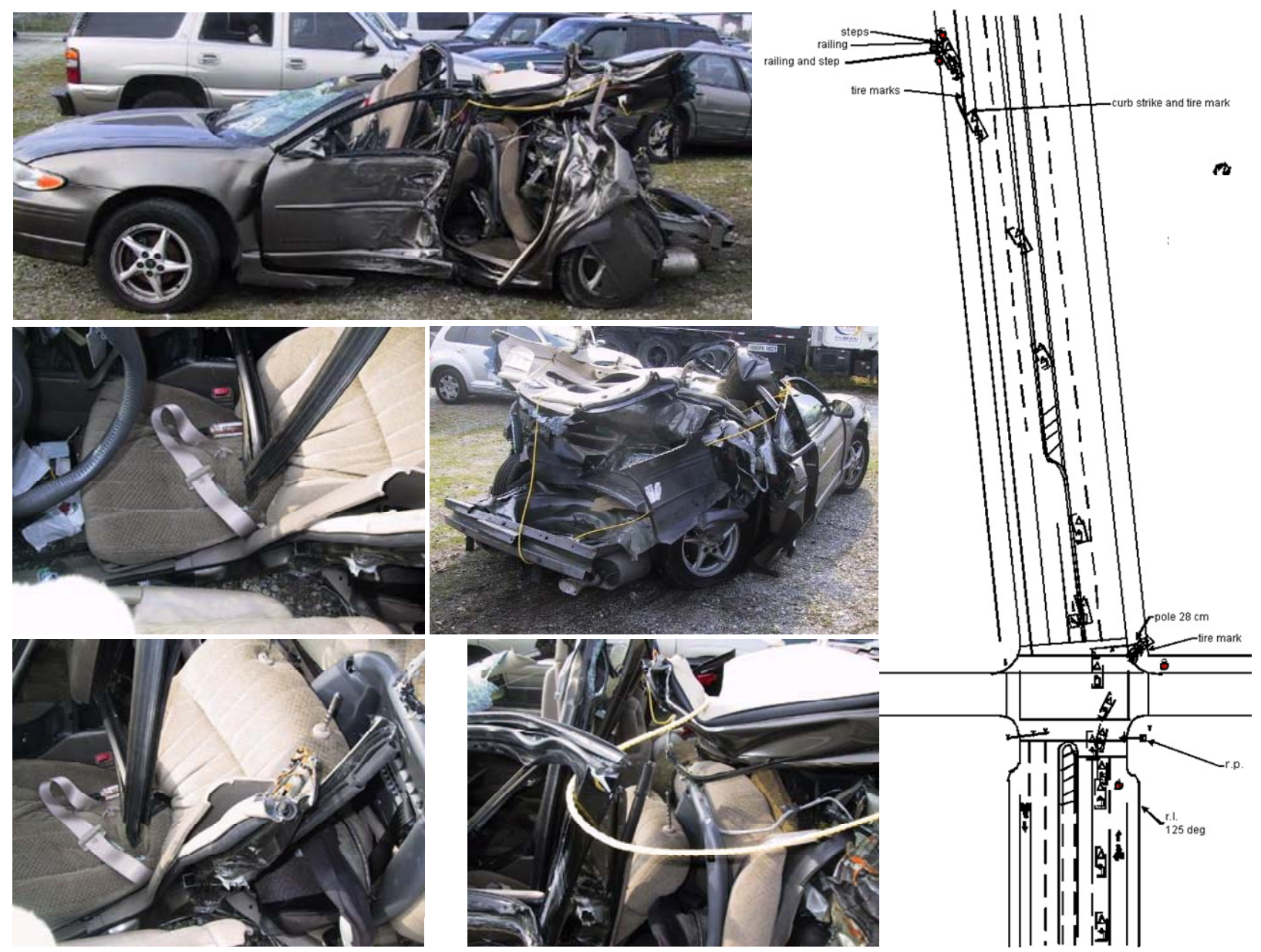

Figure A4: Vehicle path and photos for case 2003-6-99A.

2003-6-99A: Vehicle 1 was a 1997 Ford E-Series van. It was traveling east on a two way e/w four lane highway when the front contacted the rear of Vehicle 2 (case vehicle) that was in front of Vehicle 1 and stopped just prior to the intersection of a one way south highway. Vehicle 2 (case vehicle) was a 2002 Pontiac Grand Prix. Figure A4 shows the path of the Vehicle 2 and photos of the impact damage and 
interior. As a result of the contact Vehicle 2 rotated clockwise and through the intersection and the left side contacted a pole on the s/e corner of the highway before coming to rest. Vehicle 1 overrode the entire back of Vehicle 2 and then continued in an east/north direction and approximately $121 \mathrm{~m}$ (397 ft) later mounted the south curbline and the front contacted concrete steps and two metal railings of a house as it came to rest. The operator of Vehicle 1 left the area on foot. The operator of Vehicle 2 (case occupant) was extricated from the vehicle and pronounced dead at a trauma center. Both vehicles were towed.

The driver of Vehicle 2 (case occupant) was a 28 year old male, who was wearing the lap and shoulder belts. The rear impact had a PDOF of 6 o'clock and delta $V$ of $26 \mathrm{mph}$. The $2^{\text {nd }}$ impact was to the left side with a PDOF of 10 o'clock and delta $V$ of $21 \mathrm{mph}$. The driver's most severe injuries were to the chest, including lung lacerations and contusions, left hemo-pneumothorax, multiple left-side rib fractures. The NASS investigators identified the B-pillar as the source of the left-side chest injuries. Photos of the vehicle interior show deformation of the driver seat cushion and seatback with left-side damage to the vehicle from the side impact into the pole.

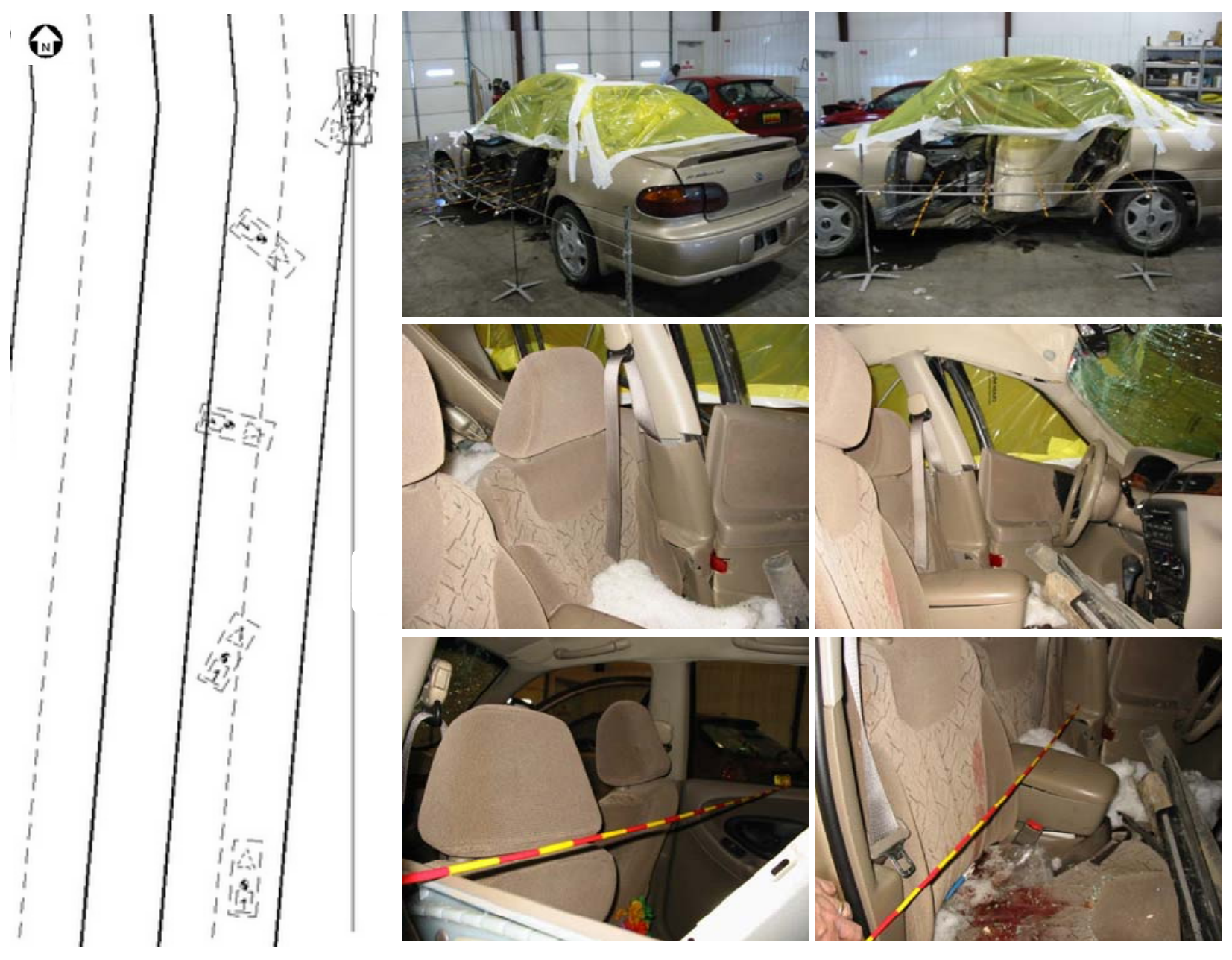

Figure A5: Vehicle path and photos for case 2004-74-14A.

2004-74-14A: Vehicle 1 (case vehicle) was a 2001 Chevy Malibu. It was north bound in lane 1 of a 4 lane, median divided, snow and ice covered, level, concrete roadway. It was daylight and clear on Sunday at 11:55. Figure A5 shows the path of Vehicle 1 and photos of the impact damage and interior. The vehicle lost control and initiated a clockwise yaw. It moved into northbound lane two as it rotated, going partially up onto the center median. It then continued to rotate, traveling northward, departed the 
right side of the roadway, facing south and impacted a metal light pole with its left side. It came to final rest approximately at the same location. The vehicle was equipped with left and right redesigned frontal airbags, which did not deploy. The vehicle was towed due to damage.

The restrained driver (case occupant) was a 21 year old female. She died in the emergency room (ER) with left lung laceration with hemomediastinum, unstable rib fractures to the left ribs 1-6, brain injuries and spleen laceration. The NASS investigator associated the injuries to impact with the left B-pillar. The collision with the pole involved the left-side driver door (GAD L). The orientation of the impact was 7 o'clock PDOF. The delta $\mathrm{V}$ was $22 \mathrm{mph}$.
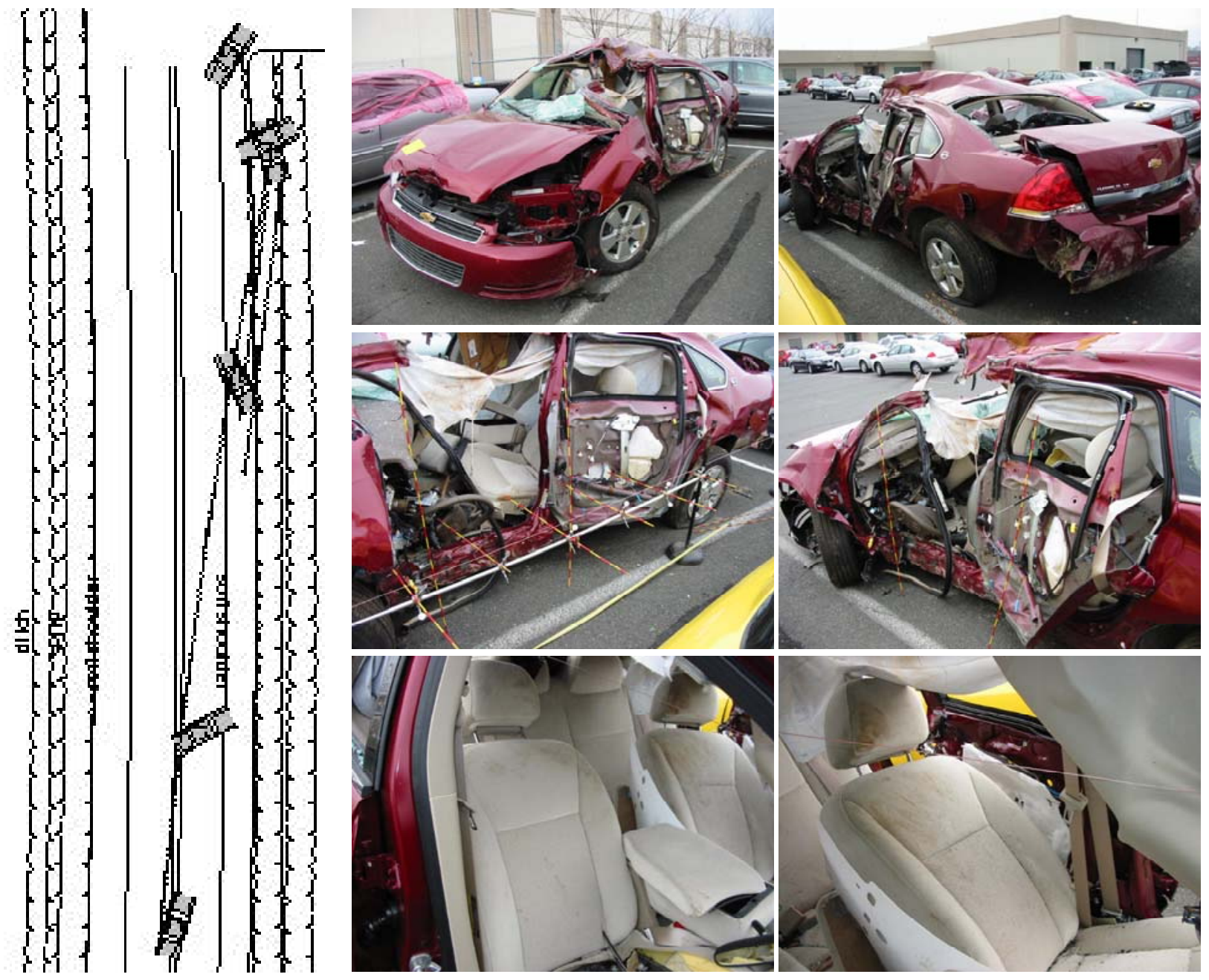

Figure A6: Vehicle path and photos for case 2005-09-185A.

2005-09-185A: V1 (Case vehicle) was a 2006 Chevrolet Impala. It was traveling south in lane one of a two lane undivided, left curve roadway. Figure A6 shows the path of Vehicle 1 and photos of the impact damage and interior. V1 lost control for unknown reasons and began to rotate clockwise as it departed the roadway to the right. V1 traveled into a ditch and struck a utility pole with its left plane. V1 continued

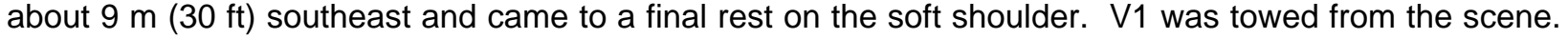
V1 contained a driver and no other occupants. The driver was transported to the hospital with fatal injuries.

The driver (case occupant) was a 30 year old male. He was restrained and died with a laceration of the heart, aortic laceration and multiple left-side rib fractures to left ribs 3-7 and right ribs 1-2. He also had a 
subarachnoid hemorrhage. The NASS investigator associated the injuries to impact with the B-pillar. The collision with the pole involved the left-side driver door (GAD L). The oriented of the impact was a 7 o'clock PDOF. The barrier equivalent speed was $21 \mathrm{mph}$. There was a subsequent right-side impact at 4 o'clock (GAD R) with an unknown delta V. 